\title{
VARIAÇÃO SAZONAL E NICTEMERAL DO ZOOPLÂNCTON NO CANAL DE SANTA CRUZ, ITAMARACÁ, PE, BRASIL
}

\author{
Fernando de Figueiredo PORTO NETO \\ Sigrid NEUMANN-LEITÃO \\ Lúcia Maria de Oliveira GUSMÃO \\ Dilma Aguiar do NASCIMENTO VIEIRA \\ Andréa Pinto SILVA \\ Tâmara Almeida e SILVA
}

Departamento de Oceanografia da UFPE

\section{RESUMO}

Estudos sobre a variação nictemeral e sazonal do zooplâncton no canal de Santa Cruz, Itamaracá - Pernambuco - Brasil, foram feitos nos períodos chuvoso e seco. Amostras foram coletadas em três estações fixas, ao longo do Canal, a cada 3 horas, num intervalo de 24 horas, em marés de sizígia, sendo utilizadas redes de plâncton com malha de $65 \mu \mathrm{m}$ de abertura, em arrastos superficiais. Dados climatológicos e hidrológicos foram obtidos para fins comparativos. O zooplâncton esteve representado por 55 taxa (9 Filos, 12 Classes e 26 Gêneros). Destacou-se Copepoda, principalmente as espécies Oithona hebes, Oithona nana, Oithona oswaldocruzi, Paracalanus crassirostris e Euterpina acutifrons. Foram também significativos: Tintinnina (Favella ehrenbergii), Foraminiferida (Cymbaloporetta atlanticus), Cirripedia (náuplios de Balanus sp), Epicaridae (larvas) e Polychaeta (larvas), além de Larvacea (Oikopleura dioica). A

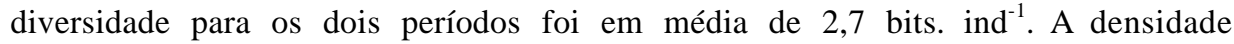
zooplanctônica variou entre $847,62 \mathrm{org} \cdot \mathrm{m}^{-3}$ a $266,063 \mathrm{org} . \mathrm{m}^{-3}$, apresentando o período chuvoso maiores quantidades. Não se observou um ciclo nictemeral regular para o zooplâncton, obtendo-se uma ampla variação entre as amostras.

Palavras-chave: Zooplâncton, Estuário, Variação nictemeral, Tropical

\section{ABSTRACT \\ Diel and Seasonal Changes in the Pelagic Copepod Community of Santa Cruz Channel, Itamaracá, Pernambuco, Brazil}

Zooplankton nictemeral and seasonal variation studies were carried out in the estuarine system of Santa Cruz Channel, Itamaracá, Pernambuco - Brazil, to assess the community structure. Sampling were carried out at three fixed stations, at 3 hours interval, during 24 hours in July/96 (rainy season) and December/96 (dry season), in spring tide. A plankton net $65 \mu \mathrm{m}$ of mesh size was hauled at surface during 3 minutes. Hydrological and climatological data were obtained for comparation with zooplankton. Fifty five zooplankton taxa (9 Phyla, 12 Classes, 26 genera) were identified. Copepoda was the most abundant outranking Oithona hebes, Oithona nana, Oithona 
oswaldocruzi, Paracalanus crassirostris and Euterpina acutifrons. Foraminiferida (Cymbaloporetta atlanticus), Tintinnina (Favella ehrenbergii), Polychaeta (larvae), Cirripedia (larvae of Balanus sp), Epicaridae (larvae) and Larvacea (Oikopleura dioica) were frequient and abundant. Species diversity average was 2.7 bits.ind ${ }^{-1}$. The density varied from 847.62org. $\mathrm{m}^{-3}$ (Bridge station, 10:00h, low tide) to 266,069org. $\mathrm{m}^{-3}$ (Igarassu station, 10:00h, low tide). Rainy season presented higher abundance. It wasn't registered a regular nictemeral zooplankton cycle, with high variations among samples.

Key words: Zooplankton, Estuary, Nictemeral variation, Tropical

\section{INTRODUÇÃO}

Nas últimas décadas, as regiões costeiras e os recursos aquáticos renováveis vêm sendo indiscriminadamente explorados. Os impactos gerados nessas áreas, oriundos de fatores diversos e baseados essencialmente em interesses econômicos imediatos e não em desenvolvimento sustentável, vêm afetando sensivelmente os mecanismos naturais (New, 1995).

Os rios que deságuam ao longo da vertente litorânea do estado de Pernambuco, apresentam crescentes sinais de desequilíbrio ecológico, tendo em vista todo o alcance das cargas poluentes a que estão submetidos, resultantes principalmente da agroindústria canavieira (FIDEM, 1975). Outro agravante é a amplitude da densidade demográfica em centros urbanos nas circunvizinhanças dos estuários. Todos estes fatores vêm ao longo dos anos causando des equilíbrios em tal ecossistema, levando a uma redução drástica nos índices de captura da pesca artesanal costeira no Estado.

Este trabalho faz parte do projeto multidisciplinar "Gerenciamento Ambiental Participativo: desenvolvimento e aplicação ao caso dos manguezais do Canal de Santa Cruz - Pernambuco", desenvolvido em conjunto pela Fundação Joaquim Nabuco de Pesquisas Sociais, Universidade Federal de Pernambuco e Universidade Federal Rural de Pernambuco. Tal projeto visa o conhecimento dos processos dinâmicos que caracterizam a ecologia dos manguezais, definindo as prioridades de conservação e recuperação de componentes ameaçados do ecossistema.

Dentro deste projeto, foram desenvolvidos estudos físico-químicos, de plâncton, necton e bentos. Nos estuários, assim como nos demais ambientes aquáticos, o zooplâncton se comporta como um dos grupos mais importantes na teia trófica, sendo responsável pela transferência aos demais elos da energia sintetizada pelo fitoplâncton (Levinton, 1995). Apresentam espécies indicadoras de qualidade de água, fornecendo subsídios sobre os processos atuantes nesses corpos d'água, uma vez que suas comunidades são diretamente influenciadas pelas condições abióticas e bióticas (Day Jr. et al., 1989; Buskey, 1993).

Este trabalho teve como objetivo conhecer a diversidade do microzooplâncton, sua distribuição espacial e temporal e correlacionar os fatores ambientais mais significativos ao zooplâncton.

\section{METODOLOGIA}


Ao longo do sistema estuarino de Itamaracá foram estabelecidas três estações fixas de coleta (Figura 1). Cada estação é representativa de um segmento distinto desse sistema, são elas:

- Estação Botafogo - na desembocadura do rio Botafogo, representando a porção norte do estuário $\left(07^{\circ} 42^{\prime} 50^{\prime \prime} \mathrm{S}\right.$ e $\left.034^{\circ} 52^{\prime} 10^{\prime \prime} \mathrm{W}\right)$;

. Estação Ponte - sob a ponte que liga a cidade de Itapissuma à Ilha de Itamaracá, na parte central do Canal de Santa Cruz (0746’30" S e 034 $53^{\prime} 40^{\prime \prime}$ W);

. Estação Igarassu - na desembocadura do rio Igarassu, próxima à barra Orange, correspondendo a porção sul da Ilha de Itamaracá (0749'20” S e 034 $\left.51^{\prime} 50^{\prime \prime} \mathrm{W}\right)$;

As amostragens foram realizadas nos meses de julho (estação chuvosa) e dezembro (estação seca) do ano de 1996, em marés de sizígia, num período de 24 horas, correspondendo a 2 ciclos diurnos consecutivos de marés (um ciclo nictemeral completo), coletando-se a cada três horas, de acordo com os picos de maré (preamar, vazante, baixa-mar e enchente). Tais coletas se deram de forma simultânea para as três estações fixas.

As coletas foram feitas através de arrastos horizontais à superfície durante 3 minutos com o barco em deslocamento lento e constante. Após as coletas, as amostras de plâncton foram colocadas em frascos plásticos com capacidade aproximada de $250 \mathrm{ml}$ e fixadas com formol a $4 \%$, neutralizado com bórax $\left(5 \mathrm{~g} .1^{-1}\right)$, de acordo com a técnica descrita por Newell e Newell (1963).

A contagem dos organismos baseou-se na menor unidade taxonômica possível de identificar-se para cada filo. Após a contagem das três sub-amostras de cada uma das amostras, calculou-se a média aritmética de ocorrência de cada organismo identificado. $\mathrm{Na}$ identificação dos organismos zooplanctônicos foram utilizadas, dentre outras, as seguintes obras: Trégouboff e Rose (1957), Björnberg (1963) e Boltovskoy (1981).

Parâmetros físico-químicos (oxigênio dissolvido, salinidade, nutrientes dissolvidos, temperatura e $\mathrm{pH}$ ) foram coletados simultaneamente. A determinação dos teores de oxigênio dissolvido se deu através da utilização do método químico de Winkler, descrito por Strickland e Parsons (1965). A determinação dos valores de salinidade baseou-se no método de Morh-Knudsen, descrito por Strickland e Parsons (1965). A determinação dos nutrientes (nitrito, nitrato, fosfato) foi feita de acordo com Strickland e Parsons (1965), o silicato-Si segundo Grasshoff et al. (1983). A concentração hidrogeniônica $(\mathrm{pH})$ foi feita através de pHmetro Beckman Zeromatic II.

O cálculo da diversidade de espécies e eqüitabilidade baseou-se em Shannon (1948). A análise multivariada dos principais taxa e parâmetros abióticos foi feita pelo coeficiente de correlação momento-produto de Pearson, após estandartização dos dados e transformação logarítmica. Foi feita análise cofenética para testar a validade dos agrupamentos. 


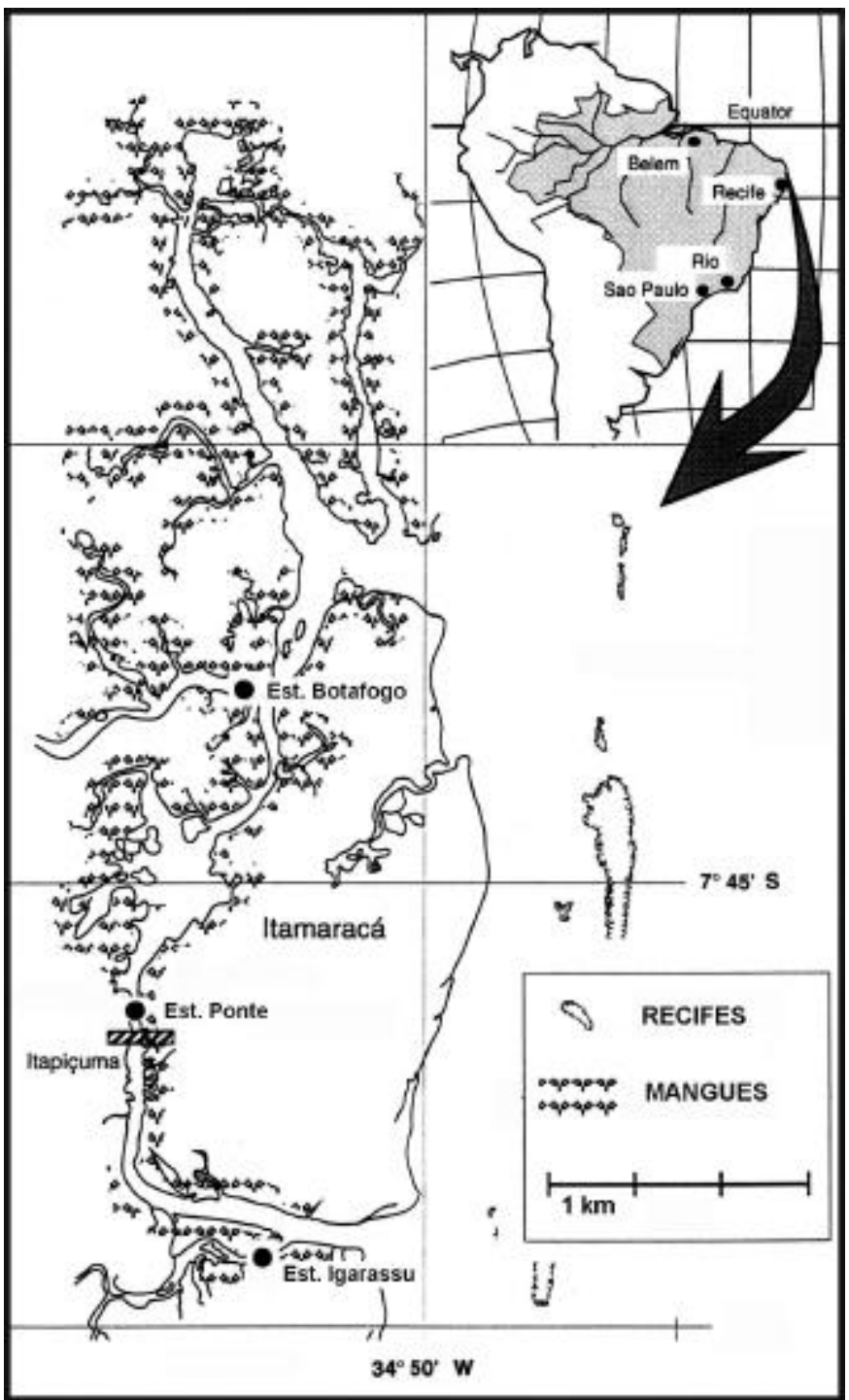

Figura 1- Região de Itamaracá (PE), com a localização das estações de coleta.

\section{RESULTADOS}

\section{Dados Abióticos}

O período chuvoso apresentou os menores índices de transparência e penetração de luz. A salinidade e temperatura estiveram, de modo geral, mais elevada em 
todas as estações no período seco (médias de $30 \%$ e $30^{\circ} \mathrm{C}$ nas estações Botafogo e Igarassu,). A estação Ponte apresentou as menores médias de salinidade (25 a 27\%o).

A variação nos teores de oxigênio dissolvido e percentual de saturação também acompanham um ritmo nictemeral definido, de acordo comas variações de maré, tendo as preamares maiores teores. Menores amplitudes de variação foram registrados na estação Ponte.

$\mathrm{O} \mathrm{pH}$ variou de neutro a alcalino, com concentrações mais altas no período chuvoso (8.2).

Nitrito e nitrato apresentaram maiores concentrações no período chuvoso, sendo a estação Botafogo a que registrou os maiores indíces (média de $0,3 \mu$ mol. $1^{-1}$ para nitrito e $1,3 \mu$ mol. $1^{-1}$ para nitrato).

Fosfato apresentou maiores concentrações no período chuvoso. O mínimo geral foi de $0,134 \mu \mathrm{mol} .1^{-1}$ na estação Botafogo, em camada intermediária, às 16:00h, preamar do período seco, e o máximo foi de $0,784 \mu \mathrm{mol} .1^{-1}$, na estação Botafogo, superfície, à 01:00h, maré enchente do período chuvoso.

O silicato em toda área estudada apresentou valor mínimo no período seco na

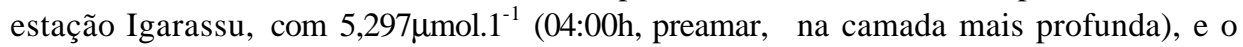
máximo no período seco na estação Botafogo, com 80,567 $\mu$ mol. $1^{-1}$ (camada intermediária, 16:00h, preamar).

\section{Zooplâncton}

O zooplâncton esteve representado por um total de 55 taxa (Tabela 01). Observou-se uma dominância do holoplâncton, em todas as estações e em todos os períodos, geralmente com abundância superior a $90 \%$ (exceto na estação Botafogo, período seco, 75\%). O meroplâncton, apresentou de 3 a 10\% de abundância (com igual exceção para a estação Botafogo, período seco, 24\%). O ticoplâncton foi o menos abundante, com percentual em torno de $1 \%$ (Fig. 2). 
Tabela 1: Densidade média dos taxa (org. $\mathrm{m}^{-3}$ ) por estação e período. $\mathrm{PC}=$ período chuvoso; $\mathrm{PS}=$ período seco

\begin{tabular}{|c|c|c|c|c|c|c|}
\hline \multirow[t]{2}{*}{ TAXA } & \multicolumn{2}{|c|}{ BOTAFOGO } & \multicolumn{2}{|c|}{ PONTE } & \multicolumn{2}{|c|}{ IGARASSU } \\
\hline & $\mathbf{P C}$ & PS & $\mathbf{P C}$ & PS & $\mathbf{P C}$ & PS \\
\hline 1. Discorbis mira & 317,21 & & & & 143,74 & \\
\hline 2. Cymbaloporetta atlanticus & 64,31 & 176,17 & & 23,12 & 1618,68 & 688,60 \\
\hline 3. Textularia $\mathrm{sp}$ & 115,46 & 83,97 & & 3,81 & 119,07 & 78,87 \\
\hline 4. Quinqueloculina $\mathrm{sp}$ & 80,03 & 52,02 & & & & 156,01 \\
\hline 5. Globorotalia $s p$ & 62,34 & 66,96 & 34,93 & 29,23 & 285,09 & 215,50 \\
\hline $\begin{array}{ll}\text { 6. } & \text { Outros Foraminiferida } \\
\text { 7. } & \text { Leprotintinnus nordqvivsti }\end{array}$ & & $\begin{array}{l}79,68 \\
64,90\end{array}$ & & & 511,04 & 97,59 \\
\hline 8. Tintinnopsis tocantinensis & 102,83 & 74,44 & 4,05 & 14.78 & & 7.74 \\
\hline 9. Favella ehrenbergii & 15,26 & 913,49 & 182,25 & 861,83 & 2678,68 & 2072,42 \\
\hline 10. Hidromedusae & 8,04 & & & & & \\
\hline 11. Nematoda & 9,62 & 9,78 & 11,56 & 42,63 & 125,92 & 154,43 \\
\hline 12. Brachionus plicatilis & 7,63 & 1,94 & 3,81 & 4,96 & 12,71 & \\
\hline 13. Trichocerca $\mathrm{sp}$ & 2727,15 & & & 1,14 & 19,70 & 7,63 \\
\hline 14. Gastropoda (véliger) & 234,04 & 1880,62 & 454,99 & 58,18 & 2526,16 & 968,74 \\
\hline 15. Bivalvia (véliger) & 329,93 & 240,98 & 5,78 & 17,56 & 96,24 & 137,63 \\
\hline 16. Polychaeta (larvas) & 4684,08 & 163,16 & 53,43 & 55,56 & 385,44 & 432,22 \\
\hline 17. Paracalanus crassirostris & & 33,88 & 566,44 & 135,71 & 5769,78 & 1802,48 \\
\hline 18. Paracalanus quasimodo & 11,44 & & 3,81 & & 14,19 & \\
\hline 19. Paracalanus indicus & 168,70 & & & & & \\
\hline 20. Temora turbinata & 22,12 & 14,34 & & & 9,53 & 42,24 \\
\hline 21. Temora sp (copepodito) & 443,39 & & & & & \\
\hline 22. Pseudodiaptomus acutus & 588,39 & 31,94 & 20,14 & 3,24 & 59,90 & 333,15 \\
\hline 23. Pseudodiaptomus richard & 582,12 & 18,09 & 18,41 & 1,92 & 179,12 & 219,61 \\
\hline 24. Acartia lilljeborgi & 336,15 & 175,49 & 609,26 & 68,48 & 265,08 & 1478,87 \\
\hline 25. Acartia sp (copepodito) & 32,82 & 205,97 & 191,89 & 90 & 1255,53 & 1202,18 \\
\hline 26. Pontellidae (náuplio) & 8653,99 & 1,14 & & & & \\
\hline 27. Oithona hebes & 541,50 & 7300,57 & 2010,10 & 2925,68 & 50323,34 & 18487 \\
\hline 28. Oithona oswaldocruzi & 397,03 & 396,38 & 452,01 & 78,20 & 1573,29 & 1169,93 \\
\hline 29. Oithona nana & 2232,52 & 150,69 & 344,00 & 73,28 & 1825,75 & 318,66 \\
\hline 30. Oithona $s p$ (copepodito) & 11,44 & 2965,26 & 1271,04 & 806,67 & 30145,38 & 24055,57 \\
\hline 31. Corycaeus speciosus & 13,35 & & & & & \\
\hline 32. Corycaeus amazonicus & 6,15 & & & & & \\
\hline 33. Darcythompsonia radans & 576,06 & 4,96 & & & & \\
\hline 34. Euterpina acutifrons & 34,20 & 86,24 & 243,56 & 46,74 & 4089,60 & 659,51 \\
\hline 35. Tigriopus $s p$ & 15,26 & 8,01 & 9,62 & 2,32 & 226,06 & 76,89 \\
\hline 36. Microsetella $s p$ (copepodito) & & & 55,91 & 2,68 & 9,53 & 32,70 \\
\hline 37. Tibes bermudensis & 84,97 & 3,43 & 3,81 & 3,81 & & \\
\hline 38. Metis $s p$ & & 38,59 & 15,55 & 3,24 & 45,117 & 28,26 \\
\hline 39. Outros Haparcticoida & 17763,44 & & 28,84 & 5,15 & & \\
\hline 40. Copepoda (outros náuplios) & 850,17 & 6802,38 & 4726,39 & 3608,08 & 48121,73 & 22063,48 \\
\hline 41. Balanus sp (náuplio) & & 3170,61 & 307,33 & 108,97 & 1604,24 & 1735,79 \\
\hline 42. Cirripedia (cypris) & 93,48 & 20,40 & & 0,38 & 77,04 & 25,81 \\
\hline 43. Epicaridae (larvas) & 24,49 & 181,14 & 77,68 & 57,59 & 419,93 & 298,75 \\
\hline 44. Decapoda (larvas) & 13,35 & 17,58 & 3,81 & 3,81 & & 6,45 \\
\hline 45. Lucifer faxoni & 7,63 & 3,05 & & & & \\
\hline 46. Porcellanidae (zoea) & 178,22 & & & & & 3,81 \\
\hline 47. Brachyura (zoea) & 14,54 & 824,16 & 13,47 & 66,05 & 160,73 & 966,91 \\
\hline 48. Cumacea & 17,97 & 3,43 & & & & \\
\hline 49. Crustacea (protozoea) & & 4,96 & & & & \\
\hline 50. Equinodermata (pluteus) & 23,06 & & & & & 13,35 \\
\hline 51. Sagitta tenuis & 132,49 & 24,73 & & 1,14 & & \\
\hline 52. Oikopleura longicauda & 660,99 & 215,82 & 337,94 & 121,52 & 9,63 & 77,97 \\
\hline 53. Oikopleura dioica & & 718,79 & 368,50 & 171,69 & 346,88 & 263,94 \\
\hline
\end{tabular}


A densidade média do período chuvoso foi de 70.300,08 org. $\mathrm{m}^{-3}$, e do período seco foi de 39.178,09org. m $^{-3}$, não tendo sido observado ciclos nictemerais regulares (Fig. 3). No período chuvoso, a biomassa nas três estações foi mais elevada do que no período seco (Fig. 4). A biomassa foi mais elevada na estação Igarassu (máximo de 682.858 org. $\mathrm{m}^{-3}$ ), onde foi encontrado muito sedimento nas amostras, indicando forte turbulência na coluna d'água.

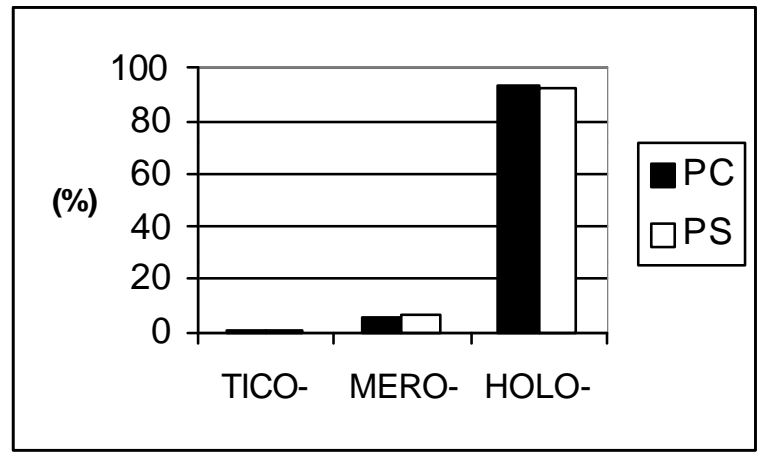

Figura 2 - Variação sazonal media do tico-mero-holoplâncton no Canal de Santa Cruz (PE).

A diversidade de espécies (Figura 5), variou de média a alta. A estação Botafogo registrou o maior e o menor valor alcançado, 3,587bits.ind ${ }^{-1}, 1,022 \mathrm{bits}_{\text {.ind }}{ }^{-1}$, respectivamente. No período chuvoso a média da eqüitabilidade (Figura 06) foi em média de 0,61. O período seco apresentou média de 0,60.

No período chuvoso, a análise cofenética apresentou um $\mathrm{r}=0,52$, não significativo. Neste período, evidenciou-se um único grupo composto por espécies estuarinas eurialinas, subdividido em 4 subgrupos (Figura 7):

Subgrupo 1: composto por náuplios de Balanus sp (Cirripedia) e copepoditos de Acartia sp.

Subgrupo 2: composto por Oithona hebes, O. nana e copepoditos de Oithona sp. Subgrupo 3: subgrupo com duas ramificações, na primeira visualiza-se Cymbaloporetta atlanticus e outros Foraminiferida, e na segunda Euterpina acutifrons, relacionando-se com a salinidade.

Subgrupo 4: composto por larvas de Polychaeta e Oithona oswaldocruzi. 


\section{Densidade - Período Chuvoso}

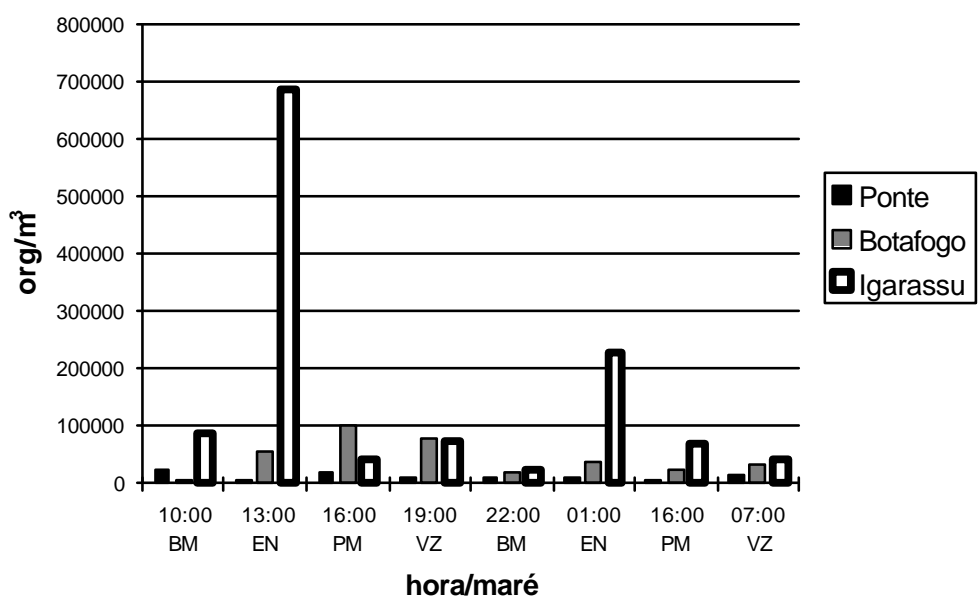

\section{Densidade - Período Seco}

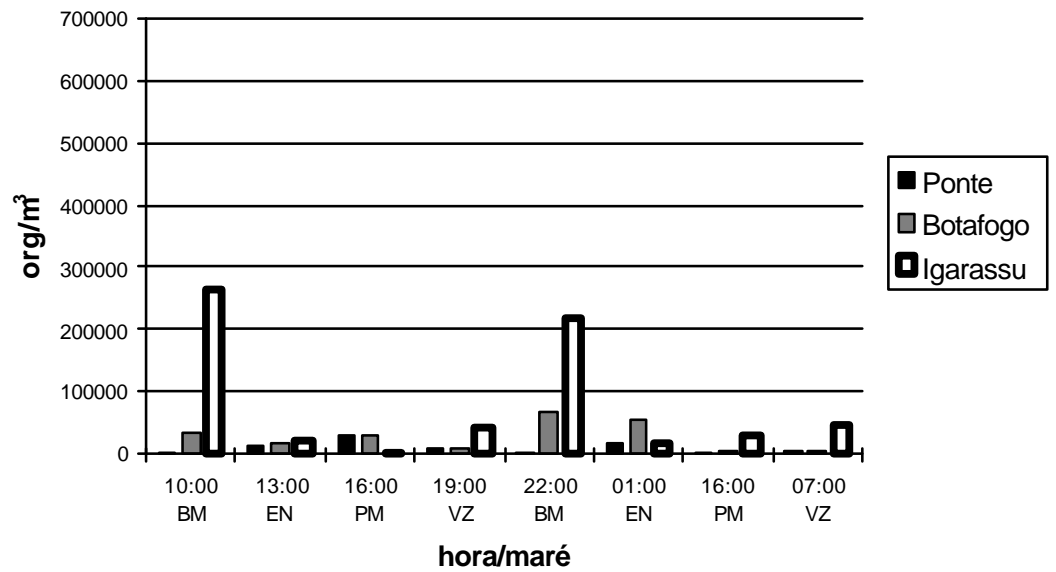

Figura 3-Variação sazonal da densidade no Canal de Santa Cruz (PE). 


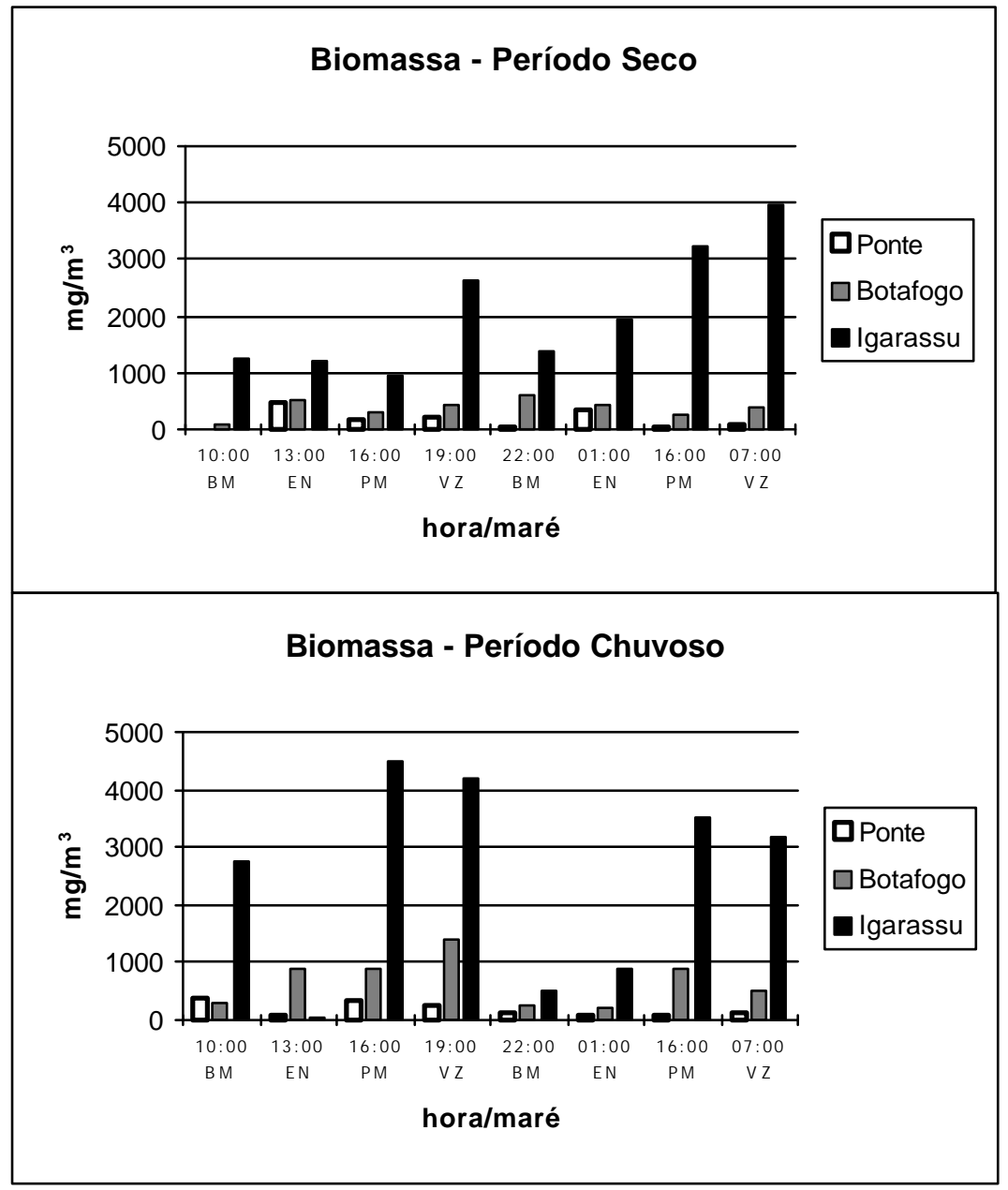

Figura 4: Variação sazonal da biomassa planctônica no Canal de Santa Cruz (PE). 


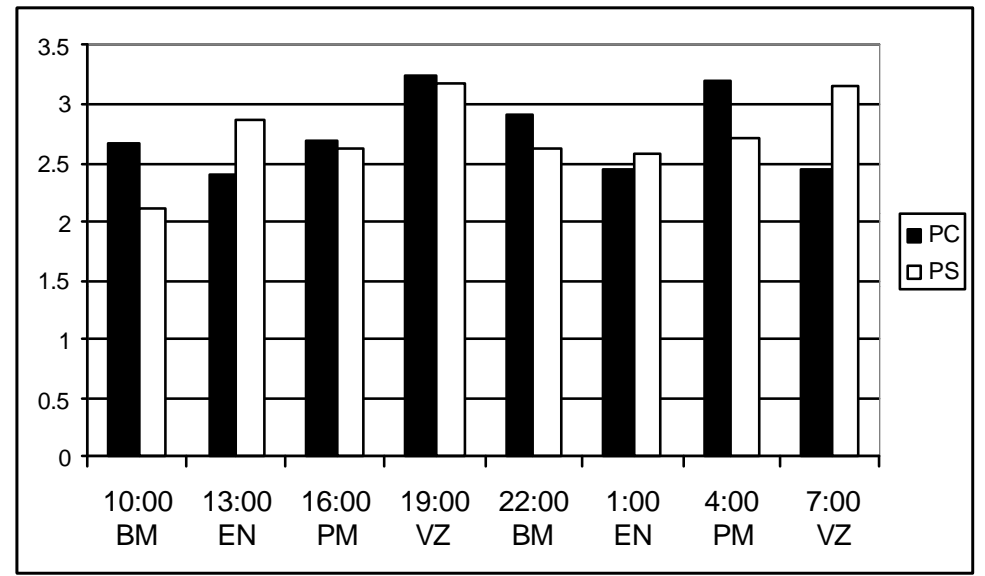

Figura 5 - Diversidade média do zooplâncton no Canal de Santa Cruz (PE).

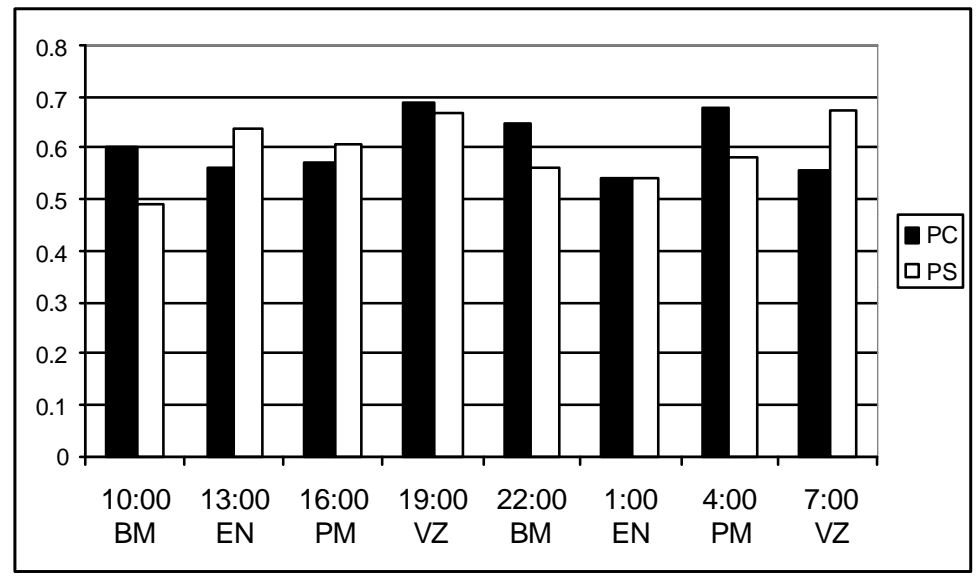

Figura 6- Eqüitabilidade média do zooplâncton no Canal de Santa Cruz (PE).

Para o período seco, a análise cofenética registrou um $r=0,64$, também pouco significativo, entretanto o dendrograma de associação de espécies (Figura 08) evidenciou 3 subgrupos principais:

Subgrupo 1: composto pela grande maioria dos taxa. Muitos são estuarinos de origem marinha (Oithona nana, Oithona hebes e Paracalanus crassirostris), além de alguns indicadores de poluição orgânica (Nematoda e larvas de Polychaeta).

Subgrupo 2: composto principalmente por taxa de origem marinha, encontrados na área costeira. 
Subgrupo 3: relaciona 2 taxa ticoplanctônicos e um de origem marinha com salinidade e oxigênio.

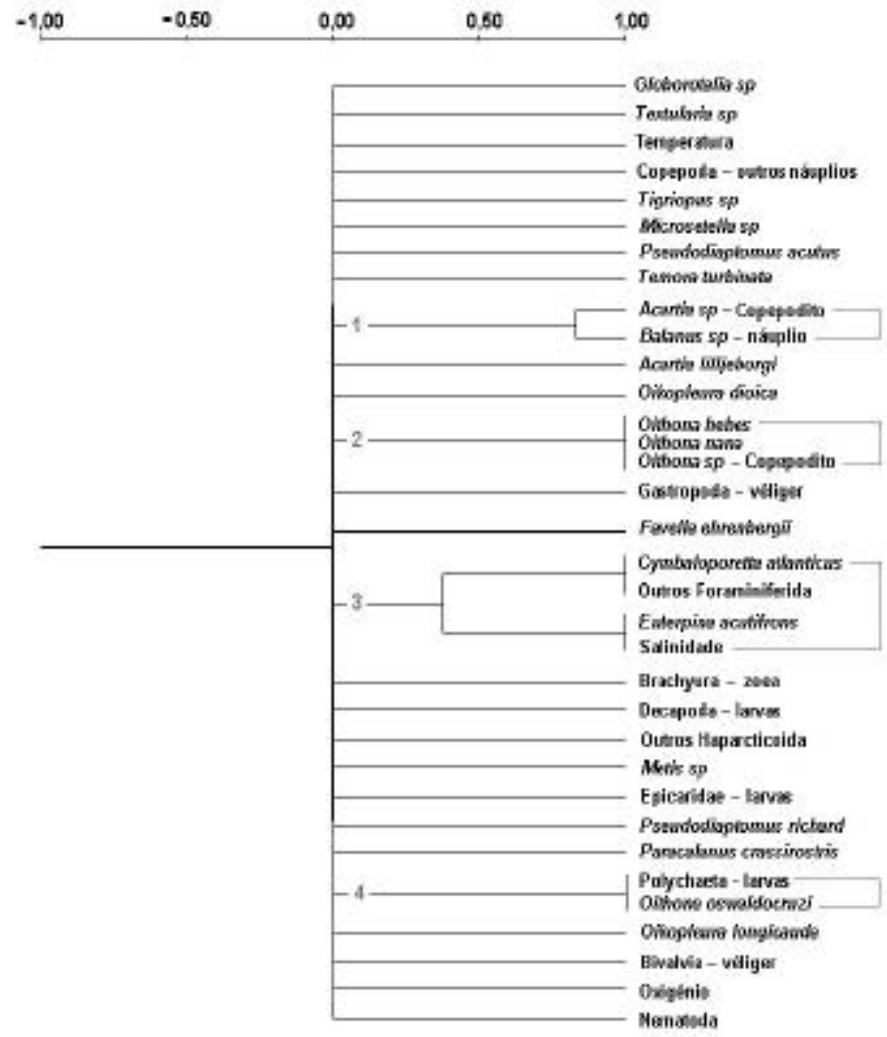

Figura 7: Associação de espécies zooplanctônicas para o período chuvoso. 


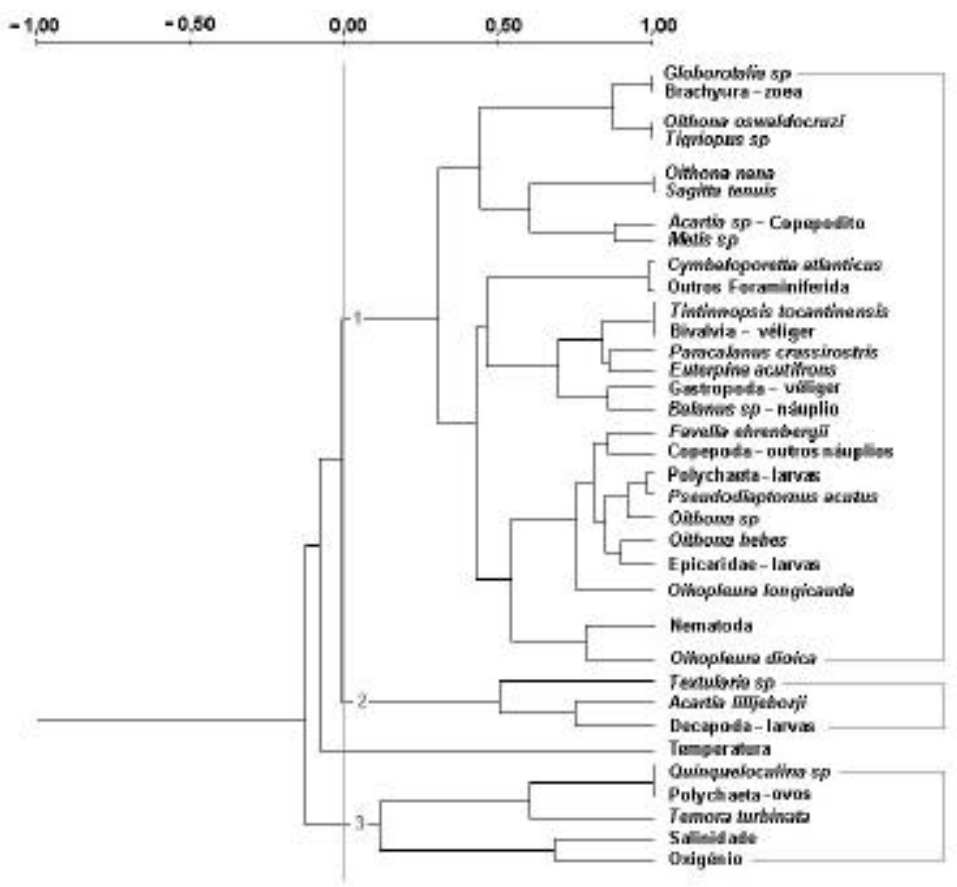

Figura 8 - Associação de espécies zooplanctônicas para o período seco.

\section{DISCUSSÃO}

Na região Nordeste do Brasil, os ciclos sazonais são definidos em duas estações climáticas: período seco, de temperaturas mais elevadas e menores taxas de pluviosidade, e um período chuvoso, onde as taxas de temperatura do ar são pouco menores e a pluviosidade aumenta. Esta diferença pode ser evidenciada no Canal de Santa Cruz através dos gradientes obtidos para os fatores climatológicos e físicoquímicos locais.

No Canal de Santa Cruz, a salinidade apresentou menores valores na superfície, aumentando de acordo com o aumento da profundidade. Todas as três estações apresentaram tendências à estratificação da salinidade, fator este que mais influencia na distribuição e abundância zooplanctônica (Rao, 1977). As maiores diferenças entre valores de salinidade nas três estações estudadas, ocorreram no estuário do rio Botafogo, com amplitude pouco maior que 5\%o. A estação Ponte apresentou a menor estratificação, certamente por localizar-se em uma região mais interna, de maior mistura da coluna d'água, fato comprovado por Medeiros (1991) e Medeiros \& Kjerfve (1993). 
O padrão sazonal e nictemeral de abundância foi bastante variável no Canal de Santa Cruz e segundo Buskey (1993) esta falta de padrão é comum a estuários tropicais e subtropicais e são importantes na estruturação da comunidade.

De uma forma geral, poucos grupos zooplanctônicos predominaram no Canal de Santa Cruz. Segundo Tundisi (1970) podem ocorrer muitas espécies no zooplâncton estuarino, mas apenas cinco ou seis constituem a maior parte da população. $\mathrm{O}$ zooplâncton estuarino caracteriza-se, geralmente, pelo predomínio de espécies holoplanctônicas (Tundisi, 1970; Day Jr et al., 1989).

A composição geral do zooplâncton no Canal de Santa Cruz foi semelhante nas duas etapas de estudo, estando representado por 55 taxa, sem grandes diferenças no ciclo nictemerais e entre os períodos seco e chuvoso, predominando Copepoda em todas as fases do ciclo de vida, fato citado para vários estuários (Tundisi, 1970; Matsumura-Tundisi, 1972; Miller, 1976; Montú, 1987; Day Junior et al. 1989). Dentre as espécies mais significativas destacaram-se Oithona hebes, Oithona nana, Oithona oswaldocruzi, Acartia lilljeborgi e Paracalanus crassirostris, somando-se aos copepoditos dos gêneros Oithona e Acartia.

Oithona hebes, é comum em águas estuarinas, sendo indicadora de áreas com manguezal, estando freqüentemente associada a Oithona oswaldocruzi (Björnberg, 1981). Neste estudo, $O$. hebes esteve mais associada com $O$. nana.

Paracalanus crassirostris, espécie, também significativa no Canal de Santa Cruz, é tida por Matsumura-Tundisi (1972) como uma espécie de grande tolerância à salinidade e temperatura, encontrada em diversas partes do mundo, porém limitada às águas costeiras de regiões tropicais e subtropicais, sendo um dos copépodos mais comumente citado em águas costeiras e estuarinas do Brasil, com exceção da lagoa dos Patos (Montú, 1980).

As espécies de Copepoda do Canal de Santa Cruz têm sido encontradas em outros estuários de Pernambuco por Paranaguá e Nascimento (1973), Paranaguá et al. (1979), Paranaguá e Koening (1980), Nascimento (1980), Nascimento e Paranaguá (1981), Por e Almeida Prado-Por (1982), Paranaguá e Nascimento-Vieira (1984) para a área estuarina de Itamaracá; Paranaguá e Gusmão(1979), Paranaguá (1985/1986) e NeumannLeitão et al. (1992; 1994) para a área estuarina de Suape; Santana-Barreto e Santos (1984) e Nascimento -Vieira e Sant'anna (1987/1989) para o estuário do rio Timbó; Paranaguá et al. (1990) e Silva (1994) para o estuário do rio Capibaribe; Nascimento - Vieira et al. (1988) e Sant'anna (1993) para a área estuarina da bacia do Pina; Santana-Barreto e Milan (1988) e Santana-Barreto et al. (1991 para o estuário do rio Formoso.

No Canal de Santa Cruz, o meroplâncton esteve constituido na sua maioria por larvas de Crustacea Decapoda, náuplios de Cirripedia e velígeres de Bivalvia e Gastropoda, algumas vezes dominando a comunidade zooplanctônica.

A presença de organismos ticoplanctônicos, representados no ecossistema presentemente estudado pelos Foraminifera, indica forte turbulência local associado à pouca profundidade; fato confirmado pela grande quantidade de sedimento nas amostras, principalmente nas do período chuvoso. 
A diversidade específica e eqüitabilidade apresentaram valores médios, indicando comunidade zooplanctônica em equilíbrio, apesar da instabilidade da área. Este equilíbrio é quebrado temporariamente, quando fluxos com poluição são trazidos dos nos Igarassu e Botafogo, onde foram observadas as mais baixas diversidades devido aos inúmeros dejetos urbanos e industriais que recebem, fato mais acentuado no período chuvoso.

O zooplâncton no Canal de Santa Cruz apresentou amplas variações quantitativas não tendo sido observado um ciclo diurno e sazonal, sendo maiores densidades registradas no período chuvoso. A falta de um padrão sazonal na abundância quantitativa parece ser mais acentuada em muitos estuários tropicais e subtropicais (Buskey, 1993), onde a atuação de fatores bióticos (predação e competição) são mais intensos.

\section{REFEERÊNCIAS BIBLIOGRÁFICAS}

BJÖRNBERG, T. K. S. On the free-living Copepods of Brazil. Bol. Inst. Oceanogr., 15(4):361-373, 1963.

BOLTOVSKOY, D. Atlas del zooplancton del Atlantico Sudoocidental y métodos de trabajos com el zooplancton marino. INIDEP, Mar del Plata. 1981, 936 p.

BUSKEY, E. J. Annual pattern of micro- and mesozooplankton abundance and biomass in a subtropical estuary. J. Plank. Res., 15(8):907-924, 1993.

DAY JR, J. W.; HALL, C. A. J.; KEMP, W. M.; YÁNÑEZ-ARANCIBIA, A. Zooplankton, the drifting consumers. In: Estuarine Ecology. Wiley-Interscience Publication. Cap. 8, p. 311- 337, 1989.

FUNDAÇÃO DE DESENVOLVIMENTO DA REGIÃO METROPOLITANA DO RECIFE (FIDEM). Levantamento preliminar da situação dos munićpios componentes da região metropolitana do Recife, Itamaracá. Recife, 1975, 132p.

GRASSHOFF, K.; ROMAM, M. R.; DEGOBBIS, D. Methods of seawater analysis. 2 ed. New York: Verlag Chemie, 1983. 317 p.

LEVINTON, J. S. Marine Biology: function, biodiversity, ecology. Oxford University Press, Oxford, 1995.420p.

MATSUMURA-TUNDISI, T. Aspectos ecológicos do zooplâncton da região lagunar de Cananéia com especial referência aos Copepoda (Crustacea). São Paulo, 1972. Tese (Doutorado), Universidade de São Paulo, Instituto de Biociências. 191 p.

MEDEIROS, C. Circulation and mixing process in the Itamaracá estuarine system, Brazil. Columbia, 1991. 131 p. (PhD Thesis). University of South Carolina.

MEDEIROS, C.; KJERFVE, B. Hidrology of a tropical estuarine system: Itamaracá, Brazil. Estuarine, Coastal and Shelf Science. London, v. 36, p. 495-515, 1993.

MILLER, C. B. The zooplankton of estuaries. In: Estuaries and enclosed seas. B. H. Kentchum (ed.). Ecosystems of the world, 26. Elsevier Scientific Publishing Company, New York. P. 103-149. 1976. 
MONTÚ, M. Síntese dos conhecimentos sobre zooplâncton estuarino. Estuário do sistema lagunar de Cananéia, complexo da Baía de Paranaguá e Lagoa dos Patos. Publicações ACIESP, 54(3):176-193. 1987.

NASCIMENTO, D. A. Composição e distribuição do zooplâncton no estuário do rio Botafogo, Itamaracá - Pernambuco - Brasil. Dissertação de Mestrado. Universidade Federal do Paraná, 1980. 108 f.

NASCIMENTO, D. A. \& PARANAGUÁ, M. N. (1981). Composição e distribuição do zooplâncton no estuário do rio Botafogo, Itamaracá - Pernambuco. ENCONTRO DE ZOOLOGIA DO NORDESTE, 3, Recife - PE. Resumo. p. 2

NASCIMENTO-VIEIRA，D. A. \& SANT'ANNA, E. E. (1987/1989). Composição do zooplâncton no estuário do Rio Timbó (Pernambuco-Brasil). Trab. Oceanogr. Univ. Fed. PE, 20:77-97.

NEUMANN-LEITÃO, S. Impactos antrópicos na comunidade zooplanctônica estuarina. Porto de Suape - Pernambuco - Brasil. Tese (Doutorado), Escola de Engenharia de São Carlos, Universidade de São Paulo, 1994. 273 p.

NEUMANN-LEITÃO, S.; GUSMÃO, L. M. O.; NASCIMENTO-VIEIRA, D. A. Zooplâncton dos estuários dos rios Massangana e Tatuoca, Suape (Pernambuco Brasil). Arq. Biol. Tecnol., 35(2): 341-360. 1992.

NEW, T. R. An Introduction to Inertebrate Conservation Biology. Oxford University Press, 1995. 194p.

NEWELL, G.H. \& NEWELL, R. Marine plankton: a preatical guide. London: Hutchinson Educat. , 1963. 221p.

PARANAGUÁ, M.N. (1985/1986). Zooplankton of the Suape area (Pernambuco-Brazil). Trab. Oceanogr. Univ. Fed. PE., 19:113-124.

PARANAGUÁ, M. N. \& GUSMÃO, L. M. O. (1979). Zooplâncton da área de Suape (Pernambuco-Brasil). Ciên. Cult., 31(7):426.

PARANAGUÁ, M. N. \& KOENING, M. L. (1980). Composição e "standing-stock" do zooplâncton dos viveiros de criação de peixes da região de Itamaracá (PE). SIMPÓSIO BRASILEIRO DE AQUICULTURA, 1, Recife, 1978. Anais..., Rio de Janeiro. p.99-107.

PARANAGUÁ, M. N. \& NASCIMENTO, D. A. (1973). Estudo do zooplâncton da região estuarina de Itamaracá. Resumo. Ciên. Cult., 25(7):198. Suplemento.

PARANAGUÁ, M.N.; NASCIMENTO, D. A.; MACEDO, S. J. (1979). Estudo Ecológico da Região de Itamaracá, Pernambuco, Brasil. II. Distribuição do zooplâncton no estuário do Rio Igarassu. Trab. Oceanogr. Univ. Fed. PE., 14:65-92.

PARANAGUÁ, M. N. \& NASCIMENTO-VIEIRA, D. A. (1984). Estudo Ecológico da Região de Itamaracá, Pernambuco-Brasil. XXV. Zooplâncton do Rio Botafogo. Trab. Oceanogr. Univ. Fed. PE, 18:193-206.

PARANAGUÁ, M. N.; SILVA, T. A.; NOGUEIRA-PARANHOS, J. D. (1990). Distribuição da comunidade zooplanctônica no estuário do Rio Capibaribe - PE. ENCONTRO BRASILEIRO DE PLÂNCTON, 4, Recife. Resumo. p. 87. 
RAO, T. S. S. Salinity and distribution of brackish warm water zooplankton in indian estuaries. In: Symposium on warm water zooplankton, Goa, 1976. Proceeding. Goa, National Institute of Oceanography, 1977. P. 196-204.

RILEY, G. A. The plankton of estuaries. In: LAUFF, G. H. (ed.) Estuaries. Washington, American Association for the Advancement of Science, 1967. p. 16-26.

SANT'ANNA, E. M. E. Estrutura e biomassa da comunidade zooplanctônica da Bacia do Pina (Pernambuco - Brasil), relacionadas com fatores ambientais. São Paulo, 1993. Dissertação de Mestrado. Escola de Engenharia de São Carlos, Universidade de São Paulo.

SANTANA-BARRETO, M. S. \& MILÁN, G. N. C. (1988). Estudo do zooplâncton no estuário do Rio Arinquidá - Rio Formoso - PE. ENCONTRO BRASILEIRO DE PLÂNCTON, 3, Pontal do Sul - PR. Resumo. p. 16.

SANTANA-BARRETO, M. S.; NOBREGA, M. N. C.; MELLO FILHO, M. T. B. (1991). Revis ão e atualização do zooplâncton no estuário do Rio Arinquidá, Rio Formoso Pernambuco. ENCONTRO BRASILEIRO DE PLÂNCTON, 4, Recife - PE, 1991. Anais..., Recife. p. 415-430.

SANTANA-BARRETO, M. S. \& SANTOS, S. M. (1984). Zooplâncton no estuário do Rio Timbó - Paulista - PE. ENCONTRO BRASILEIRO DE PLÂNCTON, 1, Arraial do Cabo - RJ. Resumo. p. 19.

SHANNON, C. E. A mathematical theory of communication. Bol. Syst. Tech. J., 27: 379423, 1948.

SILVA, T. de A. Variação nictemeral e sazonal do zooplâncton no estuário do rio Capibaribe - Recife - Pernambuco - Brasil. Recife, 1994. Dissertação (Mestrado), C. T. G., Universidade Federal de Pernambuco, Departamento de Oceanografia. 134 f.

STRICKLAND, J. D. H.; PARSONS, T. R. A manual of seawater analysis. Bull. Fish. Res. Board Can., 125:1-205., 1965.

TRÉGOUBOFF, G.; ROSE, M. Manuel de planctonologie mediterranéenne. Centre Nacional de la Recherche Scientifique, Paris. 1957, 2v.

TUNDISI, J. G. O plâncton estuarino. Contr. Avulsas Inst. Oceanogr. São Paulo, sér. Oceanogr. Biol., 19: 1-22. 1970. 